\title{
Globe
}

Revue internationale d'études québécoises

Antoine Boisclair, L'École du regard. Poésie et peinture chez

Saint-Denys Garneau, Roland Giguère et Robert Melançon, Montréal, Fides, 2009

\section{Lucie Picard}

Volume 13, numéro 1, 2010

Culture et relations internationales

URI : https://id.erudit.org/iderudit/044644ar

DOI : https://doi.org/10.7202/044644ar

Aller au sommaire du numéro

Éditeur(s)

Globe, Revue internationale d'études québécoises

ISSN

1481-5869 (imprimé)

1923-8231 (numérique)

Découvrir la revue

Citer ce compte rendu

Picard, L. (2010). Compte rendu de [Antoine Boisclair, L'École du regard. Poésie et peinture chez Saint-Denys Garneau, Roland Giguère et Robert Melançon,

Montréal, Fides, 2009]. Globe, 13(1), 167-172. https://doi.org/10.7202/044644ar d'utilisation que vous pouvez consulter en ligne.

https://apropos.erudit.org/fr/usagers/politique-dutilisation/ 


\title{
RECENSIONS
}

\author{
Frédéric Rondeau (éd.)
}

\section{Antoine Boisclair \\ L'École du regard. Poésie et peinture chez Saint-Denys \\ Garneau, Roland Giguère et Robert Melançon, Montréal, Fides, 2009.}

Ambitieux. L'ouvrage d'Antoine Boisclair - également poète, enseignant et membre fondateur des cahiers littéraires Contre-jour - est, à l'origine, une thèse de doctorat, d'où l'ampleur (remarquable) de l'effort de recherche et une structure "canonique» (une problématique, trois auteurs reconnus). Mais la visée exégétique spécifique, énoncée dans le sous-titre, ne résume pas le propos du chercheur, lequel entend offrir une synthèse du dialogue entre la poésie et la peinture au sein du champ littéraire québécois (francophone) pour la période allant de 1934 à 2004. Dans cette optique, le choix des trois œuvres analysées, basé sur leur valeur et sur l'importance de la relation au pictural pour les poètes qui les signent, répondrait également à un impératif de représentativité d'une "manière de voir " caractéristique d'un moment de l'histoire littéraire. Aussi chacun des trois volets du livre, centrés autour d'un long chapitre dédié à un auteur, comporte également un ou deux chapitres courts permettant de situer son œuvre en regard d'une tradition qu'elle dépasse (la poésie canadienne-française pour Garneau), qu'elle porte à un plein développement (les "voyants" au Québec pour Giguère; les écrits sur la peinture de Robert Marteau, Fernand Ouellette et Jacques Brault pour Melançon) ou encore qu'elle côtoie et à laquelle, dans une certaine mesure, elle s'oppose (le formalisme pour la poésie melançonienne). Un dernier mot, enfin, sur la structure du livre: j’apprécie la 
décision d'y inclure onze illustrations (œuvres des poètes-peintres, tableaux de peintres ayant influencé la démarche des poètes), tout en regrettant que l'éditeur ait fait l'économie d'une impression en couleurs.

Après avoir reparcouru l'histoire du concept de dialogue entre la poésie et la peinture en Occident (principalement) et examiné une série d'écueils épistémologiques inhérents à l'analyse des pratiques qui relèvent de ce dialogue, Boisclair élabore sa propre réflexion et sa méthodologie à partir des travaux de quelques penseurs français (notamment Maurice MerleauPonty, Jacques Rancière et Georges Didi-Huberman) qui se rattachent, de près ou de loin, à la branche esthétique de la phénoménologie. Le chercheur reprend notamment à son compte la conceptualisation par Jacques Rancière de l'ut pictura poesis propre au régime esthétique qu'inaugure la modernité: en effet, selon Rancière, la dynamique qui s'instaure du tableau (qui ne renvoie à aucun véritable langage) au texte (qui peut générer des images intérieures) met en jeu aussi bien les similitudes que les différences ontologiques entre ces deux arts. Muet et porteur d'une "distance» irréductible (Didi-Huberman), le tableau constituerait une «école du regard» pour les poètes, suscitant tantôt leur silence, tantôt leur parole. C'est dans cette perspective que Boisclair aborde les œuvres, dont son corpus principal caractérisé par la présence incontournable et polymorphe du rapport aux arts visuels: pratique de la peinture (Garneau et Giguère), écrits divers sur l'art, poèmes, auto-représentation du poète en artiste et entretiens radiophoniques avec des peintres (Melançon). Son analyse, basée sur le paramètre du regard, met clairement en évidence le rôle central et diversifié de l'ut pictura poesis dans l'élaboration et l'évolution de l'esthétique de ses auteurs. Or, il faut entendre ici le terme "esthétique» dans son acception la plus large puisqu'il englobe des "conceptions du sujet, du monde sensible et de l'expression" (p. 18) tout comme les choix qui définissent une poétique singulière. En outre, les enjeux de la relation du sujet au monde sensible, dont les poètes étudiés posent qu'il est commun à tous les humains, débordent largement les sphères artistique et littéraire: car pour l'individu comme pour les communautés, il s'agit, ultimement, d'habiter le réel. En ce sens, les créateurs président, pour eux-mêmes et pour la cité, à un "partage du sensible» (Rancière).

Si le dialogue avec la peinture constitue, pour les trois poètes étudiés, une expérience cognitive fondamentale, cette dimension est particulièrement importante chez Garneau, d'où le titre de la section qui lui est consacrée : "Apprendre à voir ". C'est d'ailleurs un rapport problématisé à l'art en tant qu'il met en jeu un sujet perfectible qui fonde, selon Boisclair, la modernité de son œuvre en regard de celle de ses prédécesseurs. Pour 
Garneau, le regard est ce lieu d'équilibre où peut advenir une rencontre de la matière et de l'esprit, une expérience de l'altérité dont l'horizon est le dépassement du dualisme corps/esprit hérité de la pensée catholique. En analysant aussi bien l'influence des discours sur l'art de son époque (au Canada français comme en France) que celle des tableaux eux-mêmes (régionalisme paysager canadien, modernité européenne), ainsi que sa réflexion sur la pratique picturale, le chercheur établit un lien entre le lyrisme critique qui est la marque distinctive de la poétique garnélienne et une expérience de l'art entendue tantôt en termes d'élan et de "chant» (Matisse), tantôt en termes de "distance» (Cézanne). L’accentuation de la dimension critique, ainsi qu'un refus anti-moderne de l'abstraction, sont mis en lien avec le silence ultime vécu par le poète comme une impasse sans remède.

Contrairement à Garneau, Roland Giguère est reconnu comme peintre tout autant que comme poète, aussi son œuvre a-t-elle bénéficié d'une attention critique sensible, dès le début, à la coprésence et à l'interaction des deux arts qu'il pratique. La poésie giguérienne, qui s'érige sans l'apport d'une réflexion critique proprement dite et caractérisée par une conscience aiguë de la matérialité du signe linguistique (d'où le terme "d'artisan» utilisé à propos de son auteur, terme que Boisclair emploie avec justesse mais qui demeure, à mes yeux, potentiellement banalisant), véhicule néanmoins une pensée esthétique que le chercheur entreprend d'expliciter dans ses grandes lignes et son évolution. Le projet giguérien, tributaire de l'esthétique surréaliste, procède d'une volonté de "donner à voir» les territoires de l'intériorité, et cela, en vue d'une recréation du visible qui permettrait, à l'individu comme à la collectivité, de "vivre mieux». La phase initiale du parcours du poète-peintre, période qualifiée par Giguère lui-même d'«âge de la parole» (1949-1960), témoigne de la confiance dans les potentialités du regard adamique dont le désir aimante la philosophie, l'art et la poésie de la première moitié du XX siècle. Riche des promesses du commencement, le regard neuf est représenté, dans les tout premiers poèmes, sous les traits d'un enfant (une influence de Garneau), puis par le type de l'homme primitif. La plongée dans l'inconscient comporte cependant des risques, que le créateur assume: on peut y rencontrer des créatures monstrueuses, plonger dans la violence du chaos, se perdre dans "la profondeur du noir". Miror, personnage inspiré du Plume d'Henri Michaux qui hante l'univers giguérien dans les années 1950, est d'ailleurs représenté comme une victime des dangers tapis dans le monde intérieur. La présence de Miror évoque également une menace plus insidieuse: s'élaborant à la frontière de la figuration et de la non-figuration, l'œuvre de Giguère est confrontée de plus en 
plus consciemment à la difficulté de voir et de figurer le monde. Les territoires et les visages sont évoqués, et non décrits; les couleurs sont traduites en mots dans des poèmes qui disent leur propre insuffisance. Une certaine perte de foi dans le langage incite alors Giguère à se tourner plus résolument vers les arts visuels, à inaugurer une nouvelle phase de sa démarche, l'«âge de l'image». Dans ses derniers recueils, le poète continue de laisser entrevoir comme possible le monde meilleur rêvé par le surréalisme, tout en s'orientant vers une poétique de l'enluminure, c'est-à-dire basée sur le travail sur "le corps de la lettre» (dont l'usage de l'anagramme constitue un exemple). Les lecteurs de Giguère se retrouveront en territoire familier dans la section que Boisclair lui a consacrée: on n'a pas l'impression ici d'une relecture profondément originale de l'œuvre (comme c'est le cas pour la section sur Garneau) ou d'une lecture largement inédite (celle de la poésie melançonienne). Plutôt, son approche lui permet de rassembler une série d'éléments connus en une perspective cohérente, ainsi que d'approfondir efficacement plusieurs aspects de l'esthétique giguérienne, souvent analysée. La validité et la flexibilité de la méthodologie du chercheur se trouvent ainsi, paradoxalement, mises en relief par l'épreuve même du "plus exploré».

Puisant à diverses époques et esthétiques, multiforme et prégnant, le dialogue avec la peinture chez Robert Melançon permet d'abord et avant tout à l'auteur d'envisager sa pratique en termes de regard («la poésie est un instrument d'optique») et de se constituer une sorte de persona intratextuelle essentiellement artistique - Boisclair convoque à ce propos le concept de transsujet élaboré par Gérard Dessons, concept d'une identité artistique qui problématiserait le rapport sujet/individu "[faisant] de l'individuation une question» (cité p. 358). C'est d'ailleurs sous le signe du questionnement de la relation entre le sujet et le visible, sur fond de silence et de blanc, que s'inaugure le parcours poétique de Melançon: «Comment accueillir ce que je vois?» Cette attention à l'extériorité sensible est le point d'ancrage d'une œuvre qui se donne pour mission de "faire voir» la réalité phénoménale telle qu'elle s'offre au regard, dans toute sa contingence, la quête de présence se situant dans la pure immanence. L'esthétique melançonienne, à cet égard, est confortée par la réflexion du poète sur la peinture du plasticien Guido Molinari. Par ailleurs, la découverte de «l'art intégré» de Mario Merola incite Melançon non seulement à faire son objet de la vie urbaine le temps d'un recueil (L'avant-printemps à Montréal, 1994), mais plus généralement à prendre un recul par rapport au monde sensible et à "encadrer l'espace» (p. 370) afin de le rendre habitable. Ce projet de transformer le visible en tableau a pour corollaire une présence de plus en plus marquée de la description dans 
les poèmes (description basée sur l'analogie et les repères spatiaux). D'autres facettes de la poétique de Melançon s'articulent également à partir de la métaphore picturale qui la fonde : ainsi, la qualité d'inachèvement (qui justifie par exemple l'existence de trois versions fortement distinctes du recueil Peinture avengle $[1979 ; 1986 ; 2005])$ renvoie à la pratique de l'esquisse (thématisée dans le recueil Le dessinateur, 2001). Dans Le paradis des apparences (2004), enfin, Melançon convoque la métaphore du trompe-l'œil et la couple à une série de stratégies textuelles qui rendent manifestes aussi bien la nature illusoire du visible que la part de plaisir inhérente à l'expérience des illusions. Accueillir, habiter, jouir du spectacle des apparences: l'esthétique melançonienne, comme le démontre Boisclair en en dessinant l'évolution, invite à un consentement au monde - le seul que nous ayons en partage.

Avant d'aller plus loin, je mentionne un malaise qui concerne la justification du corpus pris en examen. En effet, il y a un hiatus entre la structure de l'ouvrage (un axe: trois auteurs principaux pour trois époques) et la réalité du champ littéraire québécois, lequel connait, à partir des années 1980, un véritable foisonnement des relations entre les arts visuels et la poésie - et Boisclair ne manque pas de faire état de cette effervescence. D'où l'opportunité qu'il y aurait eu, à mon sens, de justifier un peu plus longuement, dès l'introduction, le choix (légitime et pertinent) de l'œuvre de Robert Melançon, non pas en tant que telle (j’insiste), mais au détriment d'autres œuvres importantes. En éclairant une sélection nécessaire (l'étude est déjà imposante), Boisclair aurait évité à certains lecteurs de traverser son ouvrage en "ressentant» l'absence de quelques grands exclus (la poésie de Denise Desautels, pour ne citer qu'un exemple).

Si l'apport des arts visuels à l'avènement puis au devenir de la poésie québécoise moderne est unanimement reconnu par la critique, il restait à articuler théoriquement cet apport d'un point de vue littéraire et à analyser le corpus dans cette perspective. L'École du regard relève brillamment le défi, Antoine Boisclair nous offrant une analyse originale et riche d'une vaste érudition, ainsi qu'une méthodologie opératoire que servent admirablement ses qualités de "lecteur de poèmes": la démarche et la poétique des auteurs en ressortent éclairées, dans le respect de l'esprit de chacune d'elle (est-ce si banal?), et efficacement contextualisées. Par ailleurs, sans jamais sacrifier la complexité de son discours, le chercheur allie à la clarté et à la précision de l'écriture une progression de l'argumentation extrêmement soucieuse de ses lecteurs. Un parti pris d'intelligibilité qui participe de la valeur d'un livre dont l'importance ne réside pas seulement dans la relecture qu'il propose de trois œuvres phares et dans les pistes qu'il ouvre à la recher- 
che, mais également - et comment éviter la référence à la thématique de Boisclair? J'y renonce... - dans sa perspective d'ensemble qui renouvelle le regard que nous posons sur le corpus national.

Dans un contexte intellectuel où la littérature est parfois considérée comme une manifestation culturelle parmi tant d'autres, on ne peut que se réjouir qu'un ouvrage de cette envergure livre une réflexion approfondie sur des enjeux esthétiques de la poésie québécoise.

Lucie Picard

Chercheure indépendante

\section{Jacques Keable}

Les folles vies de La Joute de Riopelle:

les mésaventures de l'art public,

Montréal, Lux, 2009.

Le 18 mars 2002, jour des funérailles de Jean-Paul Riopelle, un ami de l'artiste se présente devant les micros et les caméras de la télévision et, la gorge nouée par l'émotion, lui fait publiquement ses adieux devant une foule assemblée. C'est à ce moment que les Québécois apprennent, à leur grande surprise, le projet de délocaliser du quartier Hochelaga-Maisonneuve de Montréal une ouvre majeure de Riopelle, installée à proximité du Stade olympique un quart de siècle plus tôt, à l'occasion des jeux de 1976.

L'œuvre dont il est question, c'est La Joute. L'ensemble sculptural de 29 morceaux - il s'agit en fait d'une fontaine monumentale en bronze se trouve alors dans un état de détérioration avancée. Pratiquement oubliée du Musée d'art contemporain de Montréal, qui en est propriétaire au nom du ministère de la Culture, des communications et de la condition féminine, et de son hôte, la Régie des installations olympiques, La Joute est privée d'eau, partiellement rouillée et enfermée derrière des murs érigés après son installation et qui la soustraient aux regards des passants. La fontaine, dont la valeur est estimée à cinq millions de dollars malgré son état de dégradation, doit être déplacée vers un nouveau lieu, un lieu qui posera toutes sortes de défis techniques et qui occasionnera des coûts impressionnants: un nouveau square aménagé dans le Quartier international des affaires de Montréal, entre la Caisse de dépôt et placement et le Palais des congrès, sur le toit de l'autoroute Ville-Marie. 